\title{
Supplemental Oxygen Reverses Hypoxia Induced Smooth Muscle Cell Proliferation by Modulating HIF-alpha and VEGF Levels in a Rabbit Arteriovenous Fistula Model
}

\author{
Jing Wan ${ }^{2}$, Charu Lata ${ }^{1}$, Ashley Santilli ${ }^{1}$, Derrick Green ${ }^{1}$, Sabita Roy ${ }^{1}$, and Steven Santilli ${ }^{1}$ \\ ${ }^{1}$ Department of Surgery, University of Minnesota and Minneapolis Veterans Health Care System, \\ Minneapolis, Minnesota \\ 2Zhongnan Hospital Wuhan University, Wuhan, Hubei Province, China
}

\section{Abstract}

OBJECTIVES-Numerous mechanisms for the formation of intimal hyperplasia have been proposed but none have been proven or accepted. Our research focuses on the potential role of Hypoxia Inducible Factors (HIFs), VEGF, and PDGF as well as the ERK, PI3-K/AKT pathway in hypoxia mediated intimal hyperplasia processes. We hypothesize that HIF and VEGF will be down regulated with supplemental oxygen in our arteriovenous fistula rabbit model.

\begin{abstract}
METHODS—Rabbits were randomized into different experimental groups with varying oxygen exposure $\left(21 \% \mathrm{O}_{2}\right.$ or $30 \% \mathrm{O}_{2}$ ), and receipt of surgery (surgery with fistula formation, no surgery, or sham operation with skin incision only). Plasma samples were collected at designated intervals in which cytokines and smooth muscle cell proliferation were measured. In addition, cell specimens were exposed to hyperoxic, normoxic, and hypoxic environments with cytokines measured at various time points.
\end{abstract}

RESULTS-Placement of an arteriovenous fistula resulted in hypoxia induced HIF stabilization with a concurrent increase in VEGF levels. There was a 4.2-fold induction in HIF-1a levels in animals that were placed in normal air following surgery when compared to animals that were exposed to hyperoxic air. Also VEGF significantly increased post-surgery in the normoxic group, reaching a maximum VGEF level of $959 \mathrm{pg} / \mathrm{mL}$. Plasma VEGF levels in the surgery plus supplemental oxygen group were significantly lower than the normoxic surgery group with almost a $45 \%$ reduction in plasma VEGF levels $(524 \mathrm{pg} / \mathrm{mL})$. Activation of VEGF receptors on smooth muscle cells through ERK1 and AKT pathways resulted in significant smooth muscle cell proliferation and migration. These effects are dramatically reduced in animals that are exposed to a hyperoxic environment of $30 \%$ oxygen.

CONCLUSIONS-Our results suggest that short-term administration of supplemental oxygen inhibits HIFs and VEGF signaling to reduce smooth muscle proliferation in the local blood vessel. These results provide strong support for the therapeutic use of supplemental oxygen following arterial surgery to reduce intimal hyperplasia. These findings also provide a nidus for future clinical trials to determine whether this is clinical applicable in humans.

(C) 2013 Annals of Vascular Surgery Inc. Published by Elsevier Inc. All rights reserved.

Corresponding author: Steven M Santilli, MD, PhD, Division of Vascular Surgery, Department of Surgery, University of Minnesota, 420 Delaware St. SE, Mayo Mail Code 195, Minneapolis, MN, 55455, Tel: 612-625-1485. Fax: 612-626-4150. santi002@umn.edu.

Publisher's Disclaimer: This is a PDF file of an unedited manuscript that has been accepted for publication. As a service to our customers we are providing this early version of the manuscript. The manuscript will undergo copyediting, typesetting, and review of the resulting proof before it is published in its final citable form. Please note that during the production process errors may be discovered which could affect the content, and all legal disclaimers that apply to the journal pertain. 


\section{INTRODUCTION}

End-stage renal disease (ESRD) is the complete loss or almost complete loss of kidney function. In 2008, there were over 500,000 people in the US with the diagnosis of ESRD ${ }^{1}$. The number of patients with (ESRD) is expected to rise worldwide. ESRD patients who are unable to receive a kidney transplant, dialysis is required for therapy ${ }^{2}$. The Fistula First Initiative advocates that patients should have placement of an arteriovenous fistula (AVF). Unfortunately, approximately $40 \%$ of AVF are no longer functional 2 years after placement and patients require another procedure for dialysis $\mathrm{s}^{3,4,5,6}$.

It is estimated that $50 \%$ of AVF failures are due to anastomotic intimal hyperplasia (IH) ${ }^{7}$. Intimal hyperplasia is the abnormal migration and proliferation of vascular smooth muscle cells with associated deposition of extracellular connective tissue matrix which involves the endothelium and is beneath the luminal side of the internal elastic lamina ${ }^{8}$. Progression of intimal hyperplasia results in arterial occlusion and can lead to repeat operative procedures that have associated morbidity and mortality, and increased medical costs.

The effective treatment and prevention of IH in clinical practice continues to elude physicians. Treatments, including pharmacologic therapy, antioxidant therapy, heparin infusion, and gene-directed therapy of adenoviral vectors, have yet to be widely used and accepted as effective methods for preventing $\mathrm{IH}^{9,10,11,12}$.

Our laboratory has demonstrated that artery wall hypoxia is associated with both atherosclerosis and $\mathrm{IH}^{13}$. We have also now definitively shown that the short-term administration of supplemental oxygen inhibits smooth muscle cell (SMC) proliferation and prevents IH at an AVF, at the anastomotic site of arteriovenous grafts (AVG), and at the deployment site of an intra-arterial stent ${ }^{14}$.

Numerous mechanisms for the formation of IH have been proposed but none have been proven or accepted. Our investigation has focused on the role of mechanisms with oxygen occupying a central role, including hypoxia-inducible factors (HIFs), vascular endothelial growth factor (VEGF), and vascular SMC. HIFs are transcriptional regulators of genes that play a key role in the adaptation of genes to low oxygen conditions ${ }^{15,16}$. VEGF and the VEGF-receptor (R) interaction is an essential step in cell proliferation, sprouting, and migration ${ }^{17-20}$. Vascular SMC, a major cell type of the vascular wall, plays a critical role in the process of angiogenesis under both physiological and pathophysiological conditions, including wound healing ${ }^{21-24}$. VEGF induced activation of VEGF-R2 results in promotion of the ERK pathway and the PI3-K/AKT pathway in vascular EC 24 .

This manuscript focuses on the potential role of HIFs, VEGF, and platelet derived growth factors (PDGF) as well as the ERK, PI3-K/AKT pathway in hypoxia mediated IH processes. We hypothesize that HIF and VEGF will be down regulated with supplemental oxygen in our arteriovenous fistula rabbit model.

\section{METHODS}

Animal model

Fifty-two New Zealand white male rabbits, four in each group (Table 1), were assigned to one of the four following arms of the study: control with no surgery and without supplemental oxygen $(\mathrm{NS}+\mathrm{NO})$, surgery with $30 \%$ supplemental oxygen $(\mathrm{S}+\mathrm{O})$, surgery without supplemental oxygen ( $\mathrm{S}+\mathrm{NO})$, and a sham group which just received a skin incision. Each treatment group was treated for 1, 3, 7 and 21 days. Immediately after surgery the rabbits were assigned to a control cage ( $21 \%$ oxygen) or to an oxygen chamber ( $30 \%$ 
oxygen) designed and built by Plas Labs (Lansing, MI). These chambers were designed to regulate the environmental oxygen content (30\%) and relative humidity $(50 \%)$. Our standard protocols for pre-experiment care was implemented and were in accordance with an animal use protocol approved by the Minneapolis Veterans Affairs Institutional Animal Care and Use Committee ${ }^{14}$.

\section{Creation of an arteriovenous anastomosis}

After 2 weeks of acclimatization, rabbits were anesthetized with ketamine $(40 \mathrm{mg} / \mathrm{kg})$ and xylazine $(5 \mathrm{mg} / \mathrm{kg})$ intramuscularly and intubated with a $3 \mathrm{~mm}$ endotracheal tube. Anesthesia was administered with isoflurane inhalation. The right common iliac artery and vein was then exposed through a midline incision and carefully dissected. 200U/kg of intravenous heparin was administered and the common iliac artery and vein clamped proximally and distally. The iliac vein was transected and the distal end oversewn. An arteriotomy was created in the common iliac artery and the vein anastomosed in a side-to-side manner with 8-0 prolene suture. Blood loss was replaced with intravenous normal saline.

\section{Rabbit aortic cell culture}

Rabbit aortas were extracted and placed in phosphate buffered saline (PBS). The lumen was cleaned with Dulbecco's modified Eagle medium (DMEM), (Life Technologies, Grand Island, NY) solution, the smooth muscle tissue planes were cut into $1.0 \mathrm{~mm}^{3}$ pieces, and then the pieces were plated in DMEM containing 10\% fetal bovine calf serum (Life Technologies). We also cultured human umbilical vein EC with EMB-2 medium contained EGM-2 singleQuots and cultured human aorta SMC with SmBM medium contained SmGM-2 singleQuots (Lonza, Walkersville, MD).

Normoxic conditions ( $21 \%$ oxygen) are defined here as normal room air in a $5 \% \mathrm{CO}_{2}, 37^{\circ} \mathrm{C}$ cell culture incubator. To achieve hypoxia $\left(3-5 \% \mathrm{O}_{2}\right)$, cells were placed in a modular chamber (Billups Rothenberg, Del Mar, CA) and flushed with a mix of $0 \% \mathrm{O}_{2}, 5 \% \mathrm{CO}_{2}$, and $95 \% \mathrm{~N}_{2}$ at $10 \mathrm{~L} /$ minutes for 15 minutes. This method achieves $\mathrm{PO}_{2}$ levels $<35 \mathrm{mmHg}$ as determined from cell culture medium analyzed using a blood gas analyzer (Rapid Lab248; Chiron Diagnostics, Tarrytown, NY); the $\mathrm{PO}_{2}$ levels of culture supernatant from cells grown under normoxic conditions were $150-160 \mathrm{mmHg}$.

For the hypoxia treatment, cells were serum starved for 24 hour in 1\% FBS-supplemented media and then subjected to normoxia and hypoxia for different time-points. The 3-hour treatment group was used for isolation of total mRNA in cells and 24 hours for the proliferation assay, growth factor, and growth factor receptor analysis.

\section{BrdU cell proliferation assay}

The cell proliferation ELISAs measure cell proliferation by quantitating 5-Bromo-2'-deoxyuridine (BrdU) (Roche, Madison, WI) incorporated into the newly synthesized DNA of replicating cells. The rabbit aorta SMC, human umbilical vein EC and human aortic SMC cells were cultured and treated with BrdU for pulse-labeling. The cells that incorporated BrdU were subjected to immunodetection. The BrdU label in the DNA was detected using a peroxidase-conjugated anti-BrdU antibody. Each assay was repeated at least three times.

\section{Quantitative analysis of VEGF and PDGF (platelet derived growth factor) secretion}

VEGF and PDGF secretion in collected media were determined by VEGF and PDGF Immunoassay kit (R\&D Systems, Minneapolis, MN). The lower limit of the ELISA kit for VEGF and PDGF measurement was $10 \mathrm{pg} / \mathrm{ml}$. Each sample was measured in duplicate, and VEGF and PDGF concentrations were normalized by the number of cells in each sample. Each assay was repeated at least three times. 


\section{Human Angiogenesis ELISA Strip I Profiling assay}

Angiogenesis ELISA Strip I Profiling Assay (Signosis Inc, Sunnyvale, CA) allows simultaneous profiling of 8 angiogenesis cytokines; VEGF, tumor necrosis factor-alpha (TNFa), Insulin-like growth factor 1(IGF-1), Interleukin 6 (IL-6), Fibroblast Growth Factor $\mathrm{b}(\mathrm{FGFb})$, transforming growth factor- $\beta$ (TGF $\beta$ ), Epidermal Growth Factor (EGF), and Leptin.

\section{Scratch wound assay}

The rabbit aortic SMC were cultured and scratched using $10 \mu \mathrm{m}$ tips. The plates were placed into hypoxic, normoxic, and hyperoxic condition or treated with rabbit's plasma and then the BrdU assay was performed. Each assay was repeated at least three times.

\section{P GL3-VEGF-LUC plasmid transfection}

The $5^{\prime}$ upstream region of the VEGF gene from -1360 to -1126 was inserted into the plasmid pGL3 with KpnI and Bgl II sites, to obtain pGL3 VEGF-Luc. The pGL3 VEGF-Luc and pRL-TK were provided by Sundaram Ramakrishnan, PhD from the University of Minnesota. pGL3 VEGF-LUC plasmid was transferred into the rabbit aortic SMC with superfect transfection reagent (Qiagen, Valencia, CA). After 24 hours of transfection, the cells were subjected to hypoxic, normoxic, and hyperoxic conditions for 2, 4, or 6 hours. Dual-luciferase Reporter Assay System (Promega, Madison, WI) and a Luminometer were then used to test the luciferase. Each assay was repeated at least three times.

\section{Real-time Polymerase Chain Reaction (PCR)}

a. RNA isolation and cDNA preparation: For RNA preparation, RNA was isolated from cultured rabbit aortic SMC using TRIzol (Invitrogen). The complementary DNA (cDNA) was used for each PCR reaction.

b. Real-time quantitative RT-PCR: The quantification of mRNA levels was carried out using a real-time fluorescence detection method. The cDNA was prepared as described above and amplified by Applied Biosystem 7500 Fast Real-Time PCR system. Primers were designed to generate short amplification products, which spanned one intron region to detect contamination by genomic DNA for VEGF165, 121, VEGFR1 and VEGFR2. Samples from the human umbilical vein EC cDNA were used as calibrators and variations of the test gene or $18 \mathrm{~s}$ were calculated as a relative quantity compared with this group. This cell was chosen as the reference calibrator because it expressed high levels of VEGF and receptors. Results are expressed as the ratio between the gene of interest and 18s ribosomal RNA relative quantities. The results of the Real-time PCR were calculated by "Comparative Ct method of Quantitation" $(\Delta \Delta \mathrm{Ct})^{23,25}$. Each assay was repeated at least three times.

\section{Western blot analysis}

The antibodies were obtained for HIF-1a (NOVUS Biological), VEGF, Flt-1(VEGFR1), Flk-1(VEGFR2), ERK1/2 and pERK1/2(Cell Signaling Technology, Beverly, MA); AKT and pAKT, $\beta$-actin (Santa Cruz Biotechnology, Santa Cruz, CA). The density integral derived from each protein band was taken to calculate the amount in each sample. Each assay was repeated at least three times.

\section{Statistical analysis}

SPSS 11.0 software was used to analyze the data. All the continuous variables are presented as mean \pm SEM. Student's t-test was employed to assess the statistical significance of the 
difference between control and experimental treatment groups. A statistical significance was $\mathrm{p}<0.05$.

\section{RESULTS}

\section{The expression of VEGF and PDGF in the plasma}

Animals were treated as described in the Method Section and sacrificed at day 1, 3, 7 and 21. Plasma was collected after sacrifice to determine VEGF and PDGF levels with ELISA. Our results show VEGF significantly increased at days 3, 7, 21 post-surgery in the normoxic group, reaching a maximum VGEF level of $959 \mathrm{pg} / \mathrm{mL}$ on day 7. Plasma VEGF levels in the surgery plus supplemental oxygen group were significantly lower than the normoxic surgery group with almost a $45 \%$ reduction in plasma VEGF levels $(524 \mathrm{pg} / \mathrm{mL})$ (Figure 1A).

Treatment of animals that received sham surgery and subjected to normoxic or supplemental oxygen did not show any changes in plasma VEGF levels (Figure 1B). Significant increases in plasma PDGF levels were observed in animals that had surgery and supplemental oxygen, which peaked at day 3 and reached baseline levels at day 7 (Figure 1C).

\section{Human angiogenesis ELISA strip assay for profiling 7 cytokines}

We then evaluated whether surgery in the presence of normoxia or hyperoxia led to alterations in other growth factors, including TNF-alpha, IGF-1, IL-6, FGFb, TGF $\beta$, EGF, and Leptin. No significant difference was observed between the different treatment groups relative to the 7 growth factors and cytokines tested (Figures $2 \mathrm{~A}-\mathrm{G}$ ).

\section{Rabbit aortic SMC, human umbilical vein EC, and human arterial SMC proliferation using surgery with supplemental $\mathrm{O}_{2}$, surgery without supplemental $\mathrm{O}_{2}$ and sham group plasma}

Next, we determined if plasma derived from the various treatment groups modulates SMC proliferation in an ex-vivo bioassay. In these experiments, plasma from the various treatment groups was added to cultured rabbit aortic SMC for 24 hours, and BrdU was added to determine cell proliferation. A significant increase in BrdU incorporation was shown in rabbit aortic SMC treated with plasma derived from the surgery with normoxia group from all days tested (day 1-21). Plasma derived from animals that received hyperoxia showed very little SMC proliferation with levels even lower than control groups (Figure 3A). Similar effects were observed with human umbilical vein EC and human arterial SMC (Figure 3B and C).

\section{Role of VEGF-R2 in proliferative response}

To determine if the proliferative response is mediated through VEGF-R2, plasma was pretreated with the VEGF-R2 antagonist Typhostin (1uM). Our data show that Typhostin completely antagonized plasma induced BrdU incorporation indicating the role of VEGF-R2 in the observed proliferation. (Figure 4A). In contrast, treatment of plasma samples from the groups using PDGF-R antagonist did not significantly antagonize BrdU incorporation suggesting a minimal contribution of PDGF or PDGF-R in the proliferation response (Figure 4B)

\section{Surgery induces the expression of HIF-1 $\alpha$, VEGF and FLK-1 in wounded vessels which are attenuated when animals are subjected to hyperoxia}

To determine the mechanism underlying the increased SMC proliferations following surgery, anastomosed vessels were harvested at day 3, 7 and 21 and HIF-1a protein levels and VEGF-R2 levels were evaluated using Western Blot analysis. HIF-1a expression was higher in vessels harvested at day 7 and 21 in animals that received surgery and were maintained in normal air $\left(21 \% \mathrm{O}_{2}\right)$. Animals that received supplemental $\mathrm{O}_{2}$ following 
surgery showed significantly decreased HIF-1a levels (Figure 5A) with a corresponding decrease in VEGF-R2 levels (Figure 5B). Quantification of Western Blots revealed a 4.2fold induction in HIF-1a levels (Figure 5A) and 3.0-fold induction in VEGF-R2 (Figure 5B) levels at day 7 in animals that were placed in normal air following surgery when compared to animals that were exposed to hyperoxic air. Both HIF-1a and VEGF-R2 levels were significantly elevated at day $21 \mathrm{in}$ animals that received surgery without supplemental $\mathrm{O}_{2}$.

\section{Surgery induces phosphorylation of ERK-1 and AKT in wounded vessels which are attenuated when animals are subjected to hyperoxia}

To further determine the upstream mechanism underlying the increased SMC proliferation following surgery, anastomosed vessels were harvested at day 3, 7 and 21. p-ERK1/2 levels and pAKT levels were then evaluated using Western Blot analysis (Figures 6A and B). Only a single band corresponding to ERK1 was detected in the Western Blot, with a very faint ERK2 band (Figure 6A). However, the p-ERK1 band was significantly higher at all days tested in the vessels, harvested from animals that were placed in normoxic air when compared to vessels harvested from animals that were placed in hyperoxic air (Figure 6A). Similarly, p-AKT levels were also significantly greater in the vessels of animals that were placed in normoxic air (Figure 6B). To further determine the effect of hypoxia, hyperoxia, and normoxia on ERK1/2 and pAKT levels in SMC; rabbit aortic SMC were subjected to these conditions and cells lysed for the detection of p-AKT and p-ERK (Figures 6C and D). Western Blot indicated that pAKT and pERK were upregulated after 1 hour of hypoxic stress $(P<0.05 ; n=3)$, whereas the total amount of AKT and ERK remained unchanged $(P$ $>0.05 ; n=3$ ), suggesting hypoxic induction of ERK1/2 and AKT phosphorylation in rabbit aortic SMC (Figures 6C and D). Hyperoxia did not modulate ERK or AKT phosphorylation.

\section{Hypoxia induces HIF1- $\alpha$, VEGF, and VEGF receptor gene and protein expression in rabbit aortic SMC}

To further determine if hypoxia modulates HIF-1a protein levels in rabbit aortic SMC, cells were subjected to hypoxia, normoxia, and hyperoxia and nuclear extract harvested for HIF-1a protein detection using Western Blot. As expected hypoxia treatment resulted in a significant nuclear translocation of HIF-1a protein when compared to normoxic and hyperoxic treatment. Real-time PCR analysis revealed a concurrent increase in VEGF 121 and VEGF 165 mRNA (Figure 7B). Interestingly, although no significant induction in VEGF-R1 was observed under hypoxic condition (Figure 7B), a robust induction in VEGFR2 mRNA (Figure 7C) and protein levels (Figure 7D) was observed in rabbit aortic SMC that were subjected to hypoxia. To further validate that hypoxia modulates VEGF levels in rabbit aortic SMC, cells were transfected with pGL3 VEGF-Luc plasmid for 24 hours. Cells were then subjected to hypoxic, normoxic, and hyperoxic conditions for 6 hours. The expression of pGL3 VEGF-Luc was significantly higher under hypoxic condition when compared to normoxic and hyperoxic conditions (Figure 7E).

\section{Modulation of rabbit aortic SMC migration and proliferation by hypoxia, normoxia, and hyperoxia following wounding of a vessel}

To determine if hypoxia modulated rabbit aortic SMC migration and proliferation following wounding, rabbit aortic SMC were grown to confluence in glass slides. A scratch wound was inflicted using a $10 \mu \mathrm{m}$ pipette tip and the slides were placed in hypoxia, normoxia, and hyperoxia chambers for another 24 hours. Hypoxic treatment resulted in a significant increase in cell migration into the scratch wound area when compared to normoxic and hyperoxic treatment (Figure 8A and B). To determine if the increase in migration was due to an increase in cell proliferation, cells were labeled with BrdU and detected using anti BrdUFITC antibody. Although significant BrdU incorporation was observed in cells that moved 
into the scratch area, this migration was not totally a function of proliferation since some migrated cells did not show BrdU incorporation (Figure $8 \mathrm{C}$ and D). Normoxia also resulted in some migration, but both migration and proliferation were significantly lower in cells treated with hyperoxia.

\section{DISCUSSION}

Researchers in several laboratories are actively examining the causes and mechanisms of $\mathrm{IH}$. Our laboratory is the first to report in vivo evidence and mechanism that supplemental oxygen can control cellular proliferation and IH at an AVF site.

We show that creation of an AVF results in a significant induction in VEGF-A in the plasma obtained from animals exposed to ambient air 3, 7, and 21 days following placement of an AVF. Interestingly, AVF induction of VEGF was significantly attenuated in animals which were immediately subjected to $30 \%$ supplemental oxygen maintained in the hyperoxic condition for 21 days. Sham operation or placement of control animals in hyperoxia did not result in induction in VEGF.

Plasma derived from animals that had an AVF placed and were exposed to 21\% oxygen showed significant cell proliferation corresponding to the VEGF levels. Proliferation was significantly attenuated when rabbit aortic SMC, human umbilical vein EC, and human aortic SMC were exposed to plasma that was obtained from animals that were placed in hyperoxic chambers following AVF placement. These results provide strong support for our hypothesis that exposure to hyperoxia following AVF placement can significantly inhibit SMC proliferation thereby reducing IH following AVF placement.

We observed a significant increase in HIF-1 alpha stabilization and nuclear translocation in vessels harvested from animals that underwent surgery and placement in a normoxic environment. Concurrent increases in VEGF-R2 levels were also observed. Remarkably, exposure to $30 \%$ oxygen following AVF placement dramatically reduced both HIF-1a stabilization and VEGF-R2 expression in the anastomosed vessels.

The gene encoding VEGF consists of eight exons, and alternative splicing of the VEGF gene produces four different isoforms - VEGF 121, VEGF 165, VEGF 189 and VEGF 206 which contained 121, 165,189 and 206 amino acids respectively ${ }^{26,27}$. We have demonstrated for the first time that VEGF 121 and VEGF 165 isoforms are expressed under hypoxic conditions at both the mRNA level and protein level in rabbit aortic SMC. When transfected with pGL3 VEGF-Luc plasmid in rabbit aortic SMC, the hypoxia-induced transcriptional activity of VEGF was significantly autoregulated with hypoxia. This phenotypic sequence required endothelial SMC cell-cell contact, endothelial cell-derived VEGF and SMC VEGF-R2 signaling response. We found high expression of VEGF-R2 in the AVF anastomosis in 3, 7, and 21 days without oxygen, and expression on day 7, was more significant compared with the normoxia group. Blockade of VEGF-R2 with the antagonist, Tryphostin, significantly inhibited rabbit aortic SMC proliferation validating the role of VEGR-2 in the proliferative response. Furthermore, hypoxia increased VEGF-R2 expression in rabbit aortic SMC in vitro suggesting an autocrine regulation via increased levels of VEGF.

Some reports say that beta irradiation upregulates of VEGF-R2 and interacts directly with EC functions by significantly reducing their ability to differentiate and proliferate ${ }^{28}$. We proved injury also increased rabbit aortic SMC proliferation after scratch for 24 and 48 hours following a VEGF-R2 pathway in the hypoxia condition to simulate an operation on the blood vessel. Lang et al proved when blocking Raf/VEGF-R2 significantly decreased cell proliferation and migration of both endothelial cells and vascular $\mathrm{SMC}^{29}$. 
In our research, we found phosphorylation of ERK and AKT were significantly higher in vascular tissue in 3 and 7 days following AVF placement in the normoxia condition group. We speculated that VEGF combined with VEGF-R2 and activated ERK and AKT signaling enhanced the rabbit aortic SMC proliferation in vitro and in vivo. We also observed that the VEGF from rabbit plasma had the similar effects in human umbilical vein EC and human aortic SMC in the day 3 group. These results can be used as justification to proceed to a human trial in the future.

In summary, we have demonstrated placement of an AVF results in hypoxia induced HIF-1a stabilization with a concurrent increase in VEGF. Activation of VEGF-R2 on SMC through a mechanism that involved ERK1 and AKT results in significant muscle cell proliferation and migration. These effects are dramatically reduced in animals that are exposed to a hyperoxic environment. Our results suggest that the short-term administration of supplemental oxygen could inhibit HIF-1a and VEGF signaling to reduce the IH in the local blood vessel. These results provide strong support for the therapeutic use of supplemental oxygen following arterial surgery to reduce intimal hyperplasia. These findings also provide a nidus for future studies such as development of a prospective randomized trial with radiographic and clinical evaluation of anastomosis in patients with or without short-term application of supplemental oxygen to determine whether this is clinical applicable in humans.

\section{Acknowledgments}

This work was supported by NIH grant 5RO1-HL076316. We would like to thank Paul A. Silvagni DVM, PhD (W.L. Gore \& Associates) for helping us with histological slide preparation, Leslie Dickinson (Minneapolis VA Health Care System) for animal care and operative assistance, Michael Kuskowski, PhD for help with statistical analysis and Connie Lindberg for editorial assistance.

\section{References}

1. USRDS. [Assessed: March 30, 2012] Annual Data Report. United States Renal Data System Website. 2010. www.usrds.org/adr.htm

2. Centers for Disease Control and Prevention (CDC). National chronic kidney disease fact sheet: general information and national estimates on chronic kidney disease in the United States, 2010. Atlanta, GA: U.S. Department of Health and Human Services (HHS), CDC; 2010.

3. Schwab SJ. Vascular access for hemodialysis. Kidney Int. 1999; 55:2078-90. [PubMed: 10231476]

4. National Kidney Foundation. K/DOQI clinical practice guidelines for vascular access: update 2000. Am J Kidney Dis. 2001; 37(suppl 1):S137-81. [PubMed: 11229969]

5. Gibson KD, Gillen DL, Caps MT, et al. Vascular access survival and incidence of revisions: a comparison of prosthetic grafts, simple autogenous fistulas, and venous transposition fistulas from the United States Renal Data System Dialysis Morbidity and Mortality Study. J Vasc Surg. 2001; 34:694-700. [PubMed: 11668326]

6. National Kidney Foundation. NKF-K/DOQI clinical practice guidelines for vascular access: update 2006. Am J Kidney Dis. 2006; 48 (suppl 1):S176-276. [PubMed: 16813989]

7. Parthasarathy S, Santanam N, Ramachandran S, Meilhac O. Oxidants and antioxidants in atherogenesis-An appraisal. J Lipid Res. 1999; 40:2143-57. [PubMed: 10588940]

8. Davies MG, Hagen PO. Pathobiology of intimal hyperplasia. Br J Surg. 1994; 81:1254-69. [PubMed: 7953384]

9. Clowes AW, Reidy MA. Prevention of stenosis after vascular reconstruction: pharmacologic control of intimal hyperplasia—a review. J Vasc Surg. 1991; 13:885-91. [PubMed: 2038110]

10. Davies MG, Dalen H, Austerheim AM, Gulbrandsen TF, Svendsen E, Hagen PO. Suppression of intimal hyperplasia in experimental vein grafts by oral 1-arginine supplementation and single ex vivo immersion in deferoxamine manganese. J Vasc Surg. 1996; 23:410-20. [PubMed: 8601882] 
11. Chen C, Lumsden AB, Hanson SR. Local infusion of heparin reduces anastomotic neointimal hyperplasia in aortoiliac expanded polytetrafluoroethylene bypass grafts in baboons. J Vasc Surg. 2000; 31:354-63. [PubMed: 10664503]

12. Kibbe MR, Nie S, Yoneyama T, Hatakeymna K, Lizonova A, Kovesdi I, et al. Optimization of ex vivo inducible nitric oxide synthase gene transfer to vein grafts. Surgery. 1999; 126:323-9. [PubMed: 10455901]

13. Lee ES, Bauer GE, Caldwell MP, Santilli SM. Association of artery wall hypoxia and cellular proliferation at a vascular anastomosis. J Surg Res. 2000; 91:32-7. [PubMed: 10816346]

14. Lee ES, Caldwell MP, Tretinyak AS, Santilli SM. Supplemental oxygen controls cellular proliferation and anastomotic intimal hyperplasia at a vascular graft-to-artery anastomosis in the rabbit. J Vasc Surg. 2001; 33(3):608-13. [PubMed: 11241134]

15. Osada-Oka M, Ikeda T, Imaoka S, Akiba S, Sato T. VEGF-enhanced proliferation under hypoxia by an autocrine mechanism in human vascular smooth muscle cells. J Atheroscler Thromb. 2008 Feb; 15(1):26-33. [PubMed: 18270456]

16. Lauzier MC, Robitaille GA, Chan DA, Giaccia AJ, Richard DE. BiPS, a matrix metalloprotease inhibitor, is a novel and potent activator of hypoxia-inducible factors. Mol Pharmacol. $2008 \mathrm{Jul}$; 74(1):282-8. [PubMed: 18424552]

17. Karamysheva AF. Mechanisms of Angiogenesis. Biochemistry. 2008; 73:935-48.

18. Li WW, Talcott KE, Zhai AW, Kruger EA, Li VW. The role of therapeutic angiogenesis in tissue repair and regeneration. Adv Skin Wound Care. 2005 Nov-Dec;18(9):491-500. quiz 501-2. [PubMed: 16365547]

19. Roy H, Bhardwaj S, Yla-Herttuala S. Biology of vascular endothelial growth factors. FEBS Lett. 2006 May 22; 580(12):2879-87. [PubMed: 16631753]

20. Mukherjee S, Tessema M, Wandinger-Ness A. Vesicular trafficking of tyrosine kinase receptors and associated proteins in the regulation of signaling and vascular function. Circ Res. 2006 Mar 31; 98(6):743-56. Review. [PubMed: 16574915]

21. Olsson AK, Dimberg A, Kreuger J, Claesson-Welsh L. VEGF receptor signalling: In control of vascular function. Nat Rev Mol Cell Biol. 2006; 7:359-71. [PubMed: 16633338]

22. Shibuya M. Vascular endothelial growth factor receptor-1 (VEGFR-1/ Flt-1): A dual regulator for angiogenesis. Angiogenesis. 2006; 9:225-30. [PubMed: 17109193]

23. Banerjee S, Mehta S, Haque I, Sengupta K, Dhar K, Kambhampati S, Van Veldhuizen PJ, Banerjee SK. VEGF-A165 induces human aortic smooth muscle cell migration by activating neuropilin-1VEGFR1-PI3K axis. Biochemistry. 2008; 18:47(11):3345-51. [PubMed: 18284215]

24. Adya R, Tan BK, Punn A, Chen J, Randeva HS. Visfatin induces human endothelial VEGF and MMP-2/9 production via MAPK and PI3K/Akt signalling pathways: novel insights into visfatininduced angiogenesis. Cardiovasc Res. 2008 May 1; 78(2):356-65. [PubMed: 18093986]

25. Sivanesan S, How TV, Bakran A. Sites of stenosis in AV fistulae for haemodialysis access. Nephrol Dial Transplant. 1999 Jan; 14(1):118-20. [PubMed: 10052489]

26. Houck KA, Ferrara N, Winer J, Cachianes G, Li B, Leung DW. The vascular endothelial growth factor family: identification of a fourth molecular species and characterization of alternative splicing of RNA. Mol Endocrinol. 1991; 5(12):1806-14. [PubMed: 1791831]

27. Tischer E, Mitchell R, Hartman T, Silva M, Gospodarowicz D, Fiddes JC, Abraham JA. The human gene for vascular endothelial growth factor. Multiple protein forms are encoded through alternative exon splicing. J Biol Chem. 1991; 266(18):11947-54. [PubMed: 1711045]

28. Kermani P, Leclerc G, Martel R, Fareh J. Effect of ionizing radiation on thymidine uptake, differentiation, and VEGFR2 receptor expression in endothelial cells: the role of VEGF(165). Int J Radiat Oncol Biol Phys. 2001 May 1; 50(1):213-20. [PubMed: 11316566]

29. Lang SA, Schachtschneider P, Moser C, Mori A, Hackl C, Gaumann A, Batt D, Schlitt HJ, Geissler EK, Stoeltzing O. Dual targeting of Raf and VEGF receptor 2 reduces growth and metastasis of pancreatic cancer through direct effects on tumor cells, endothelial cells, and pericytes. Mol Cancer Ther. 2008 Nov; 7(11):3509-18. [PubMed: 19001434] 
A

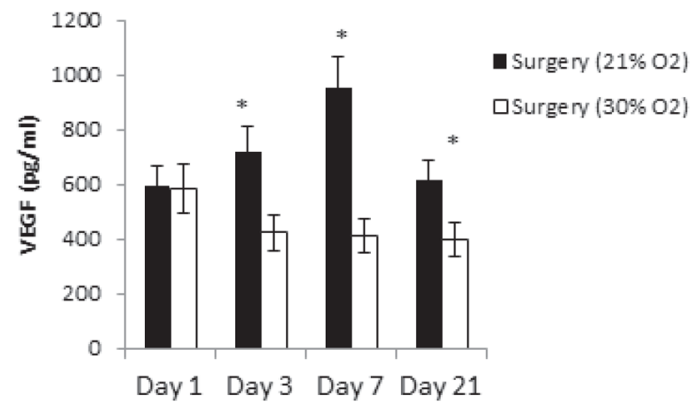

$B$

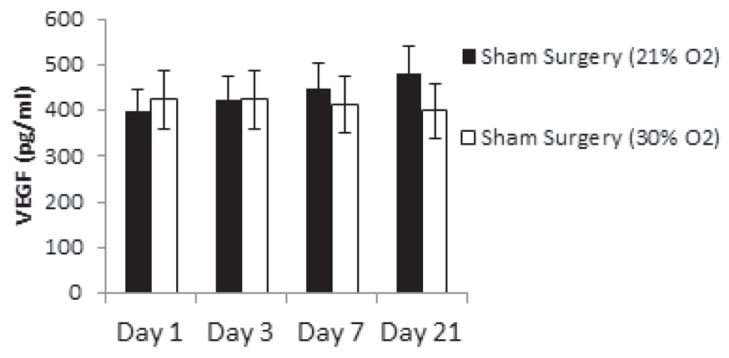

\section{C}

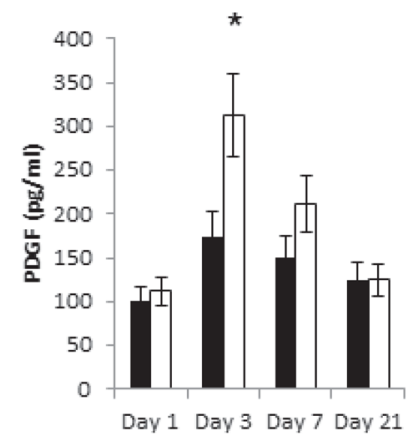

- Surgery $(21 \% 02)$

口Surgery (30\%02)

Figure 1. Plasma expression of VEGF and PDGF in animal treated with supplemental oxygen New Zealand white rabbits, four in each group, were assigned to one of the four following arms of the study: Animals underwent A) Surgery and was exposed to normoxia (Surgery $21 \%$ ) or to supplemental oxygen (Surgery 30\%). B) A second group of animal underwent sham surgery (Skin incision) and were exposed to normoxia (sham surgery $21 \%$ ) or $30 \%$ supplemental oxygen (Sham surgery 30\%). Each treatment group was treated for 1, 3, 7 and 21 days. Plasma was collected after sacrifice to determine A) VEGF and B) and C) PDGF levels with ELISA. $* \mathrm{P}<0.05$. Each group had 4 animals. 
Growth factors

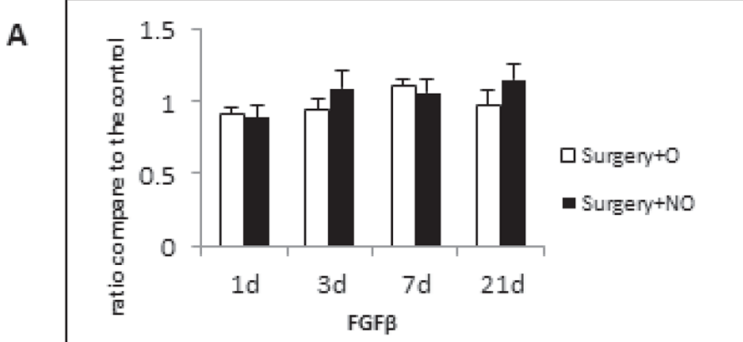

B

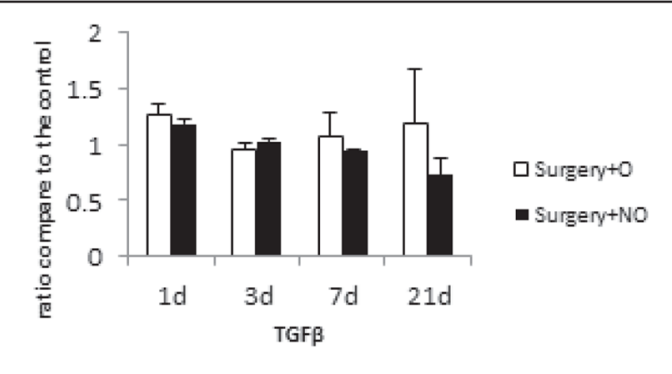

C

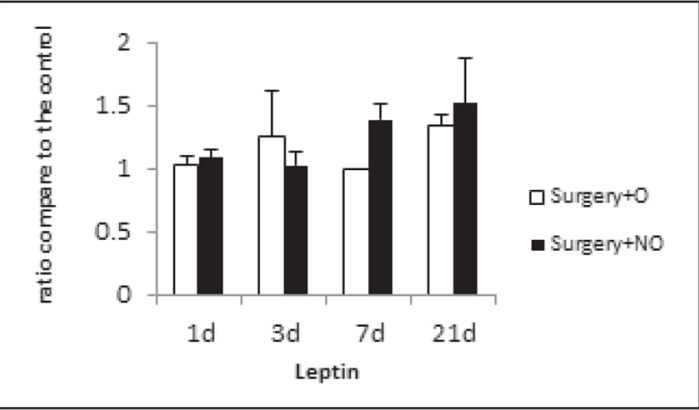

D

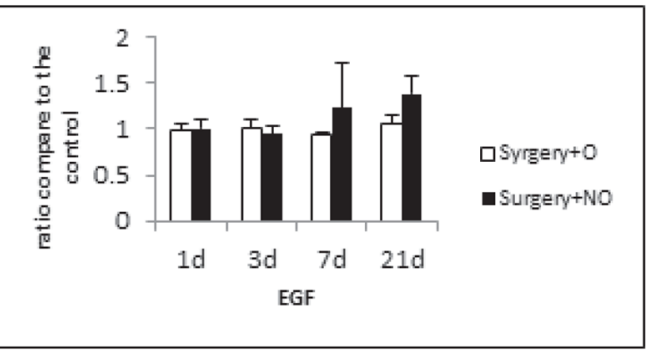

E

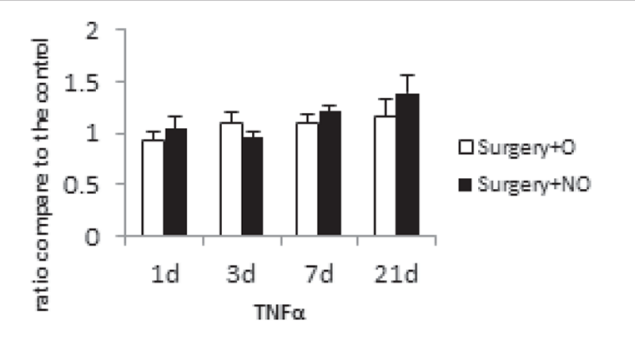

$\mathbf{F}$

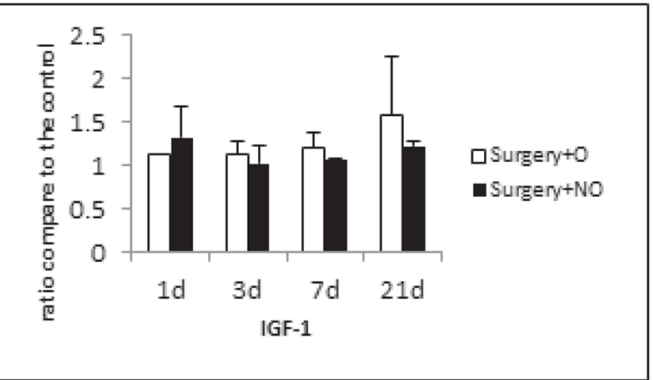

G

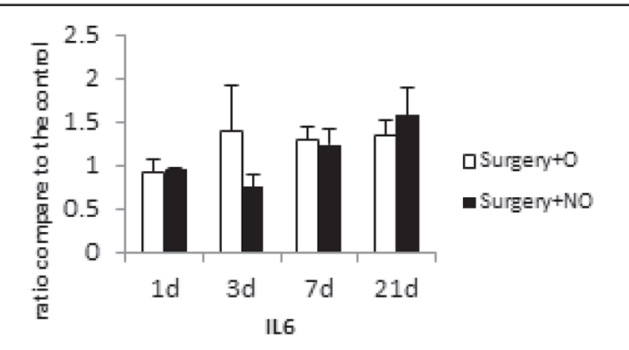

Figure 2. Plasma expression of cytokines in animal treated with supplemental oxygen

Human angiogenesis ELISA strip assay was used for profiling seven cytokines. Surgery+O (30\%) groups plasma are represented by white bars and Surgery+NO $(20 \%)$ are represented by black bars. All test results are compared with that of control plasma and expressed as percent of control. There was no difference in the seven cytokines: TNFa, IGF-1, IL-6, $\mathrm{FGFb}$, TGFb, EGF, and Leptin, with or without oxygen. 
A

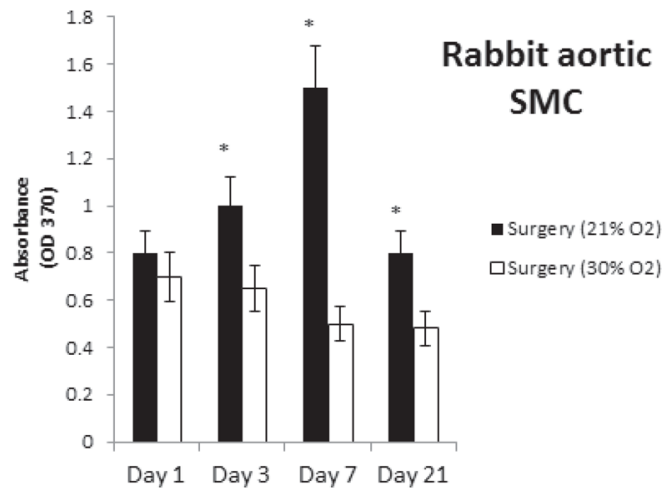

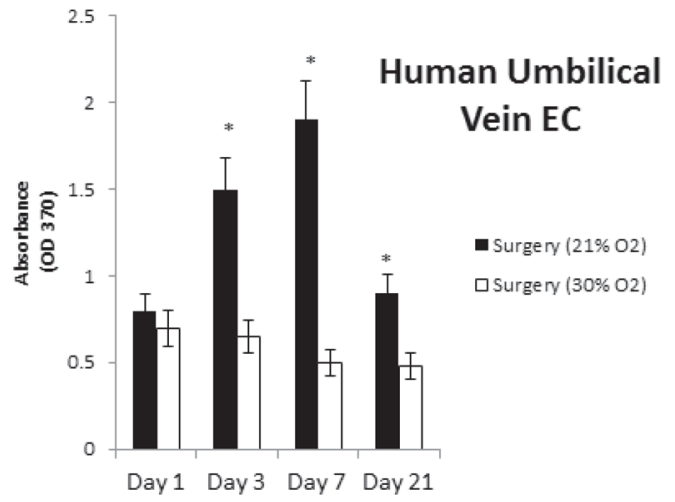

C

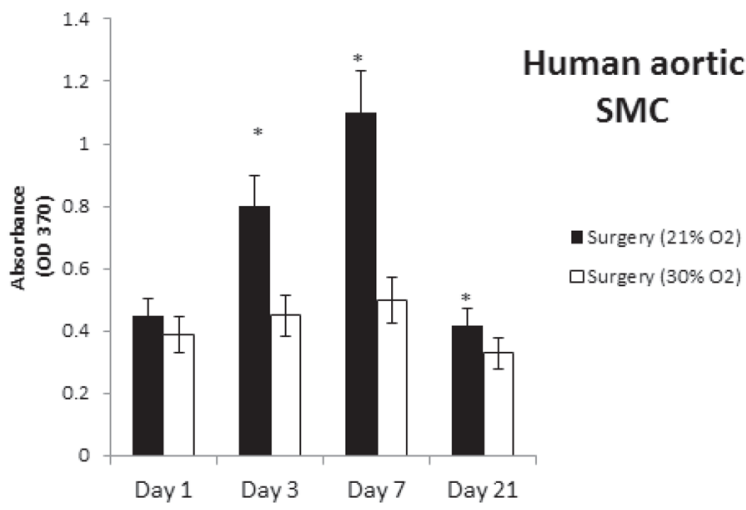

Figure 3. SMC proliferation using plasma from animals with surgery and supplemental O2, surgery without supplemental $\mathrm{O2}$ and sham group

Plasma from the various treatment groups was added to either A) cultured rabbit aortic SMC B) human umbilical vein EC and C) human arterial SMC for 24 hours, and BrdU added to determine cell proliferation. Each data point represent mean of 4 separate experiment \pm standard deviation. $* \mathrm{P}<0.05$. 

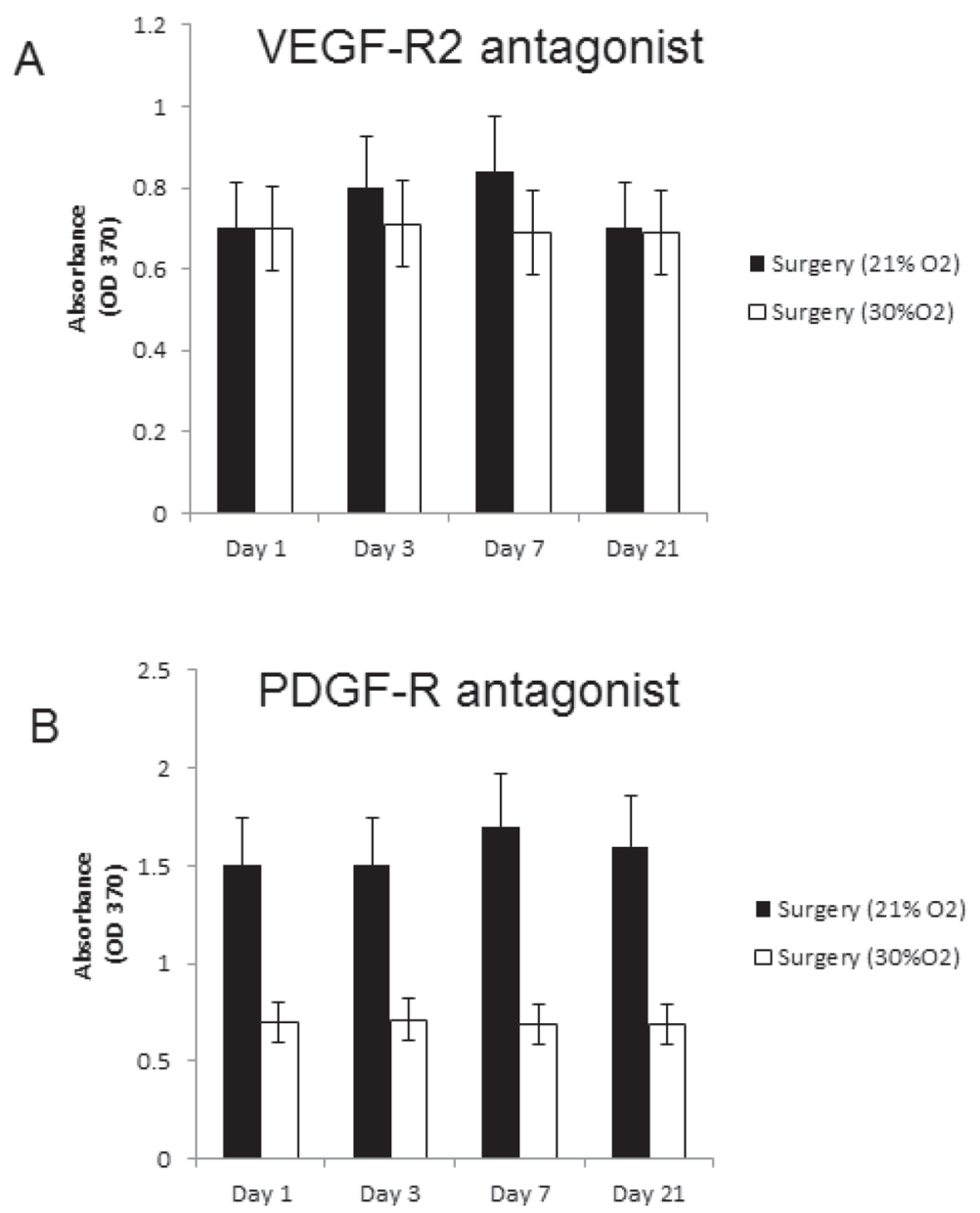

Figure 4. Role of VEGF-R2 and PDGF receptor in SMC proliferative response Plasma from normoxic and hyperoxia treated animals were pretreated with A) VEGF-R2 antagonist Typhostin (1uM) and B) PDGF-R antagonist (1uM). BrdU incorporation was used to determine proliferation. Each data point represent mean of 4 separate experiment \pm standard deviation. $* \mathrm{P}<0.05$. 

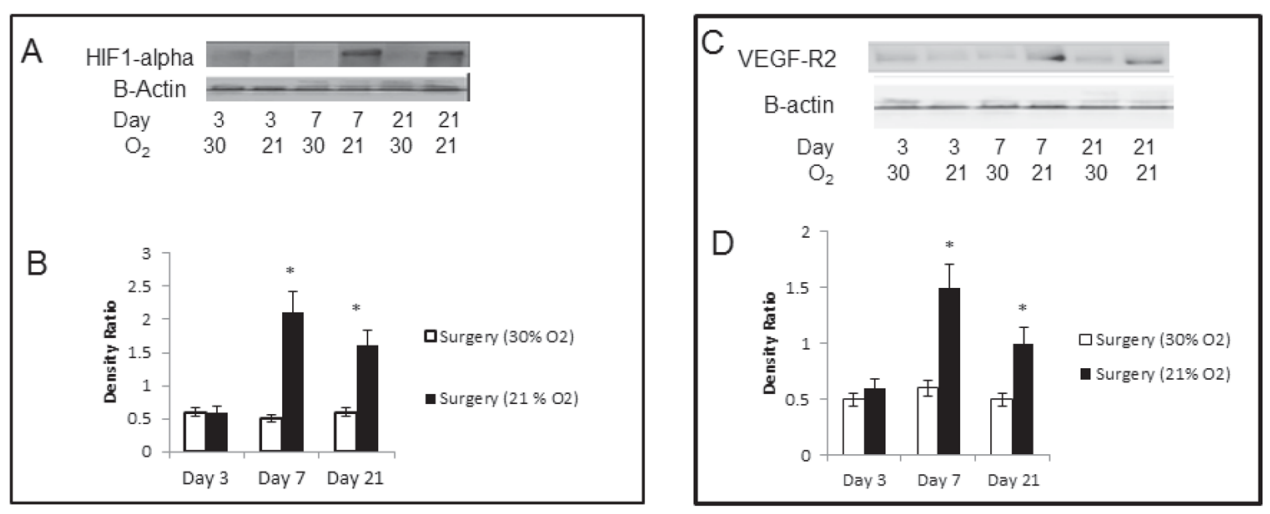

Figure 5. Surgery induces the expression of HIF-1a, VEGF and FLK-1 in wounded vessels which are attenuated when animals are subjected to hyperoxia

The expression of HIF-1a, VEGF and FLK-1 in wounded tissue was determined. A and B) HIF-1a expression using western blot analysis $C$ and D) VEGF expression using western blot analysis. Histograms (B and D) data represents mean of 4 separate experiment \pm standard deviation. $* \mathrm{P}<0.05$. 

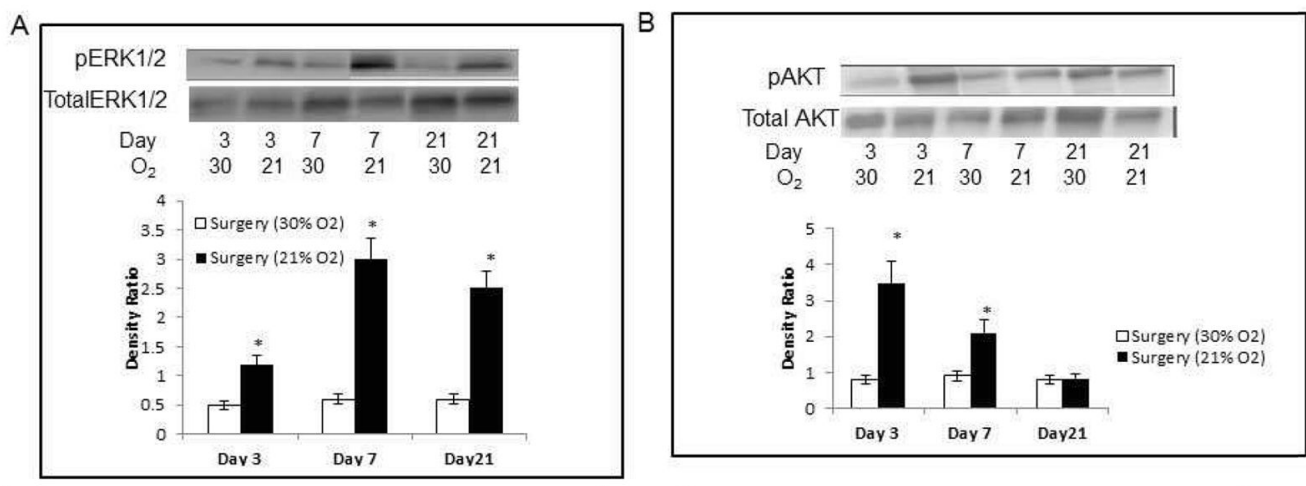

C
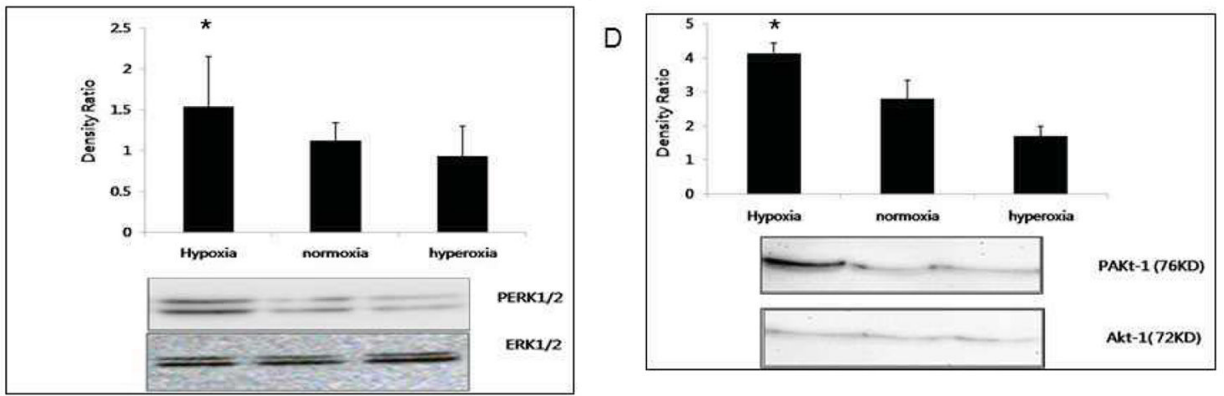

Figure 6. Surgery induces phosphorylation of ERK1 and AKT in wounded vessels which are attenuated when animals are subjected to hyperoxia

The expression of ERK1/2 and AKT in all the tissue from wounded area and rabbit aortic SMC were determined using western blot analysis. A) p-ERK1/2 protein level in 3, 7, and 21 days tissue treated with hyperoxia (30\%) or normoxia (21\%). B) pAKT protein level in 3, 7, and 21 day tissue treated with hyperoxia (30\%) or normoxia (21\%). C) p-ERK1/2 in rabbit aortic SMC treated with hypoxia, normoxia, and hyperoxia. $(\mathrm{P}<0.05)$. D) p-AKT expression in rabbit aortic SMC treated with hypoxia, normoxia, and hyperoxia. Data represents mean of four separate experiment \pm standard deviation. $* \mathrm{P}<0.05$. 


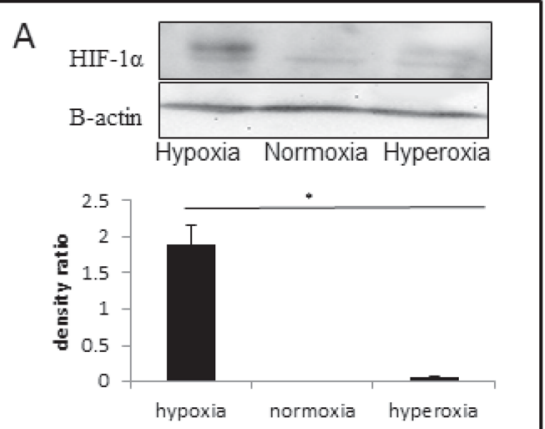

C

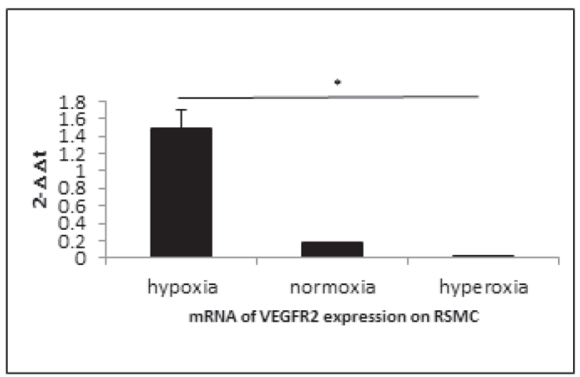

$B$

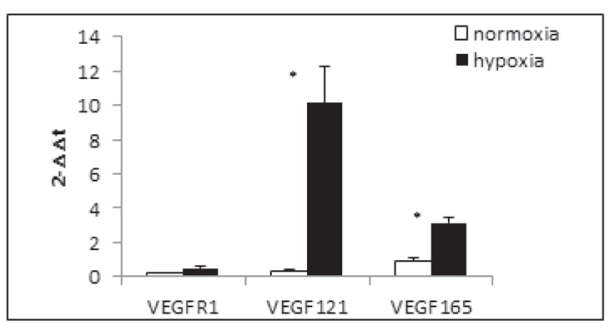

D

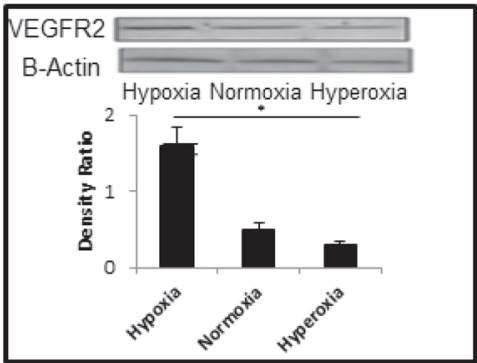

E

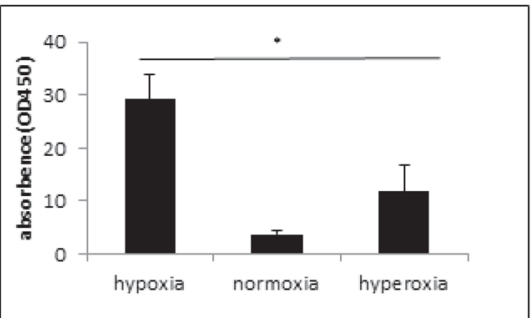

Figure 7. Hypoxia induces HIF1-a, VEGF, and VEGF receptor gene and protein expression in rabbit aortic SMC

A) HIF-1 alpha expression in rabbit aortic SMC treated with hypoxia, normoxia, and hyperoxia. B) Real time PCR expression of VEGF-R1, VEGF-165 and VEGF-121 in rabbit aortic SMC treated with hypoxia, normoxia, and hyperoxia. C) Real time PCR expression of VEGF-R2 mRNA level in rabbit aortic SMC. D) Western blot analysis of VEGF-R2 mRNA level in rabbit aortic SMC. E) SMC cells were transfected with pGL3 VEGF-Luc plasmid for 24 hours. Cells were then subjected to hypoxic, normoxic, and hyperoxic conditions for 6 hours. The expression of pGL3 VEGF-Luc determined and normalized to renilla luciferase to equalize for transfection efficiency. Data represents mean of 4 separate experiment \pm standard deviation. $* \mathrm{P}<0.05$ 


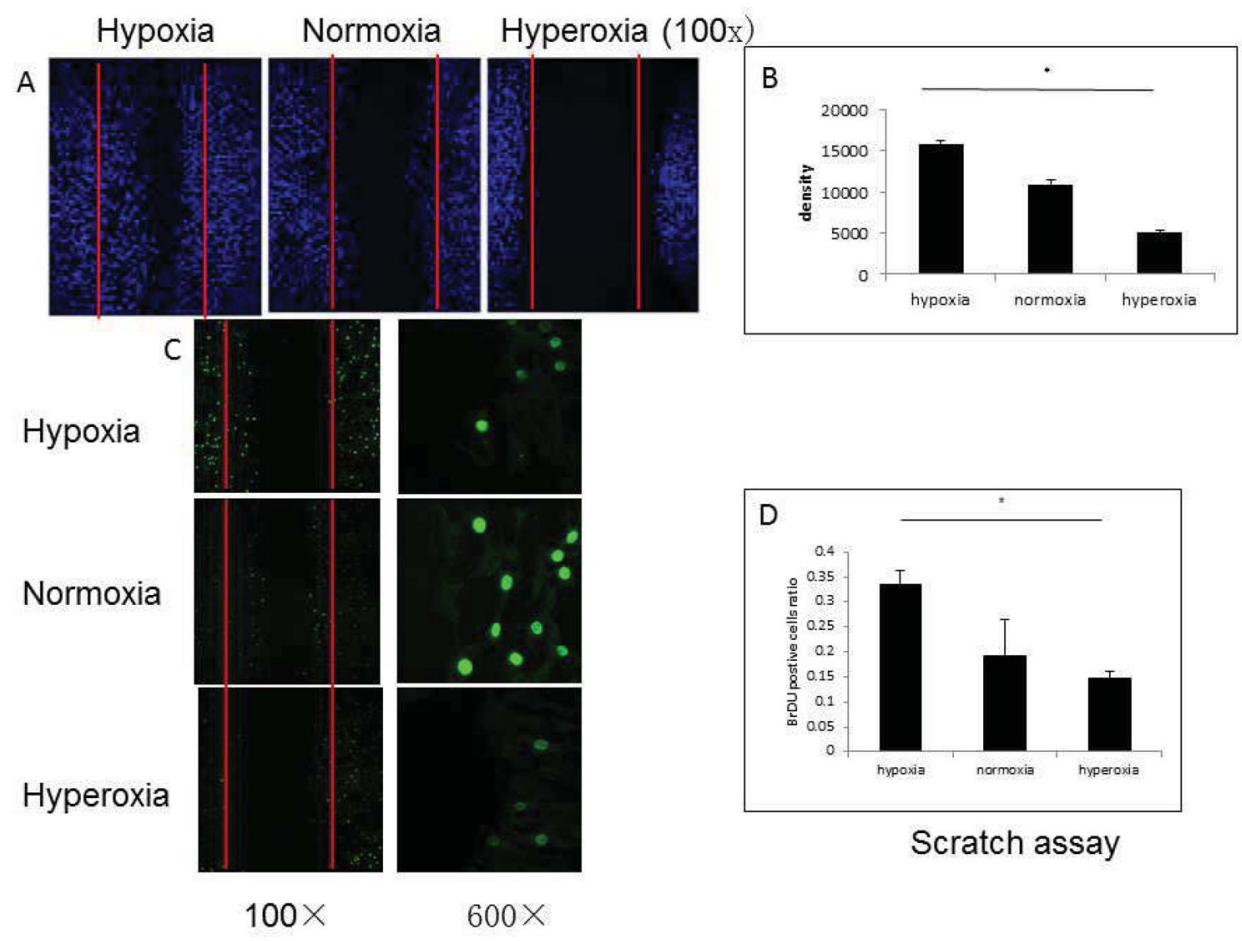

Figure 8. Modulation of rabbit aortic SMC migration and proliferation by hypoxia, normoxia, and hyperoxia following scratch wounding

A) Cells were cultured on slides, and after 24 hours scratched with pipette tips. The slides were placed in hypoxic, normoxic (20\%), and hyperoxic (30\%) chambers for another 24 hours. DAPI was used to stain the nucleus (x100). B) Quantification of cell number migrated to wound sites. C) BrDU immunofluorescence assay. Scratched slides were placed under hypoxia, normoxia, and hyperoxia and labeled with BrDU fluorencence antibody. D) Quantification of BrDU positive cells $(* \mathrm{P}<0.05)$. 


\section{Grouping of Rabbits}

Table 1

Fifty-two rabbits were divided with only four being in the control group, then the other forty-eight rabbits were divided with four rabbits in each of the other category (surgery with $\mathrm{O} 2$, surgery without $\mathrm{O} 2$, and sham surgery) that were sacrificed at four different time intervals.

\begin{tabular}{|l|l|l|l|l|}
\hline Groups & Day $\mathbf{1}$ & Day $\mathbf{3}$ & Day $\mathbf{7}$ & Day $\mathbf{2 1}$ \\
\hline Control & 4 rabbits & & & \\
\hline Surgery with $\mathbf{0 2}$ & 4 rabbits & 4 rabbits & 4 rabbits & 4 rabbits \\
\hline Surgery without $\mathbf{0 2}$ & 4 rabbits & 4 rabbits & 4 rabbits & 4 rabbits \\
\hline Sham & 4 rabbits & 4 rabbits & 4 rabbits & 4 rabbits \\
\hline
\end{tabular}

